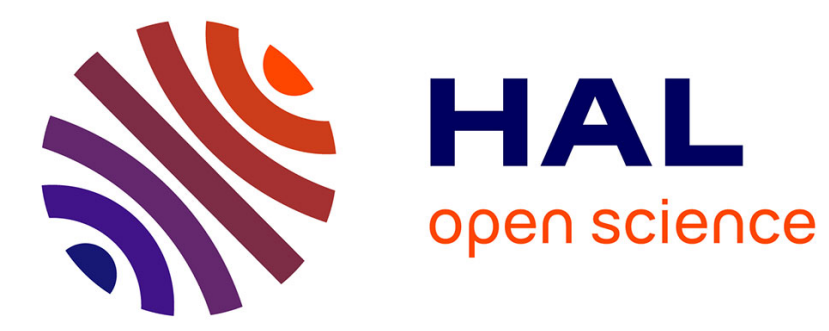

\title{
Travailler dans un village urbain : pratiques et représentations spatiales des travailleurs créatifs \\ Basile Michel
}

\section{To cite this version:}

Basile Michel. Travailler dans un village urbain : pratiques et représentations spatiales des travailleurs créatifs. Norois, 2017, 242, pp.39-54. 10.4000/norois.6042 . hal-01649837

\section{HAL Id: hal-01649837 \\ https://hal.science/hal-01649837}

Submitted on 6 Jan 2020

HAL is a multi-disciplinary open access archive for the deposit and dissemination of scientific research documents, whether they are published or not. The documents may come from teaching and research institutions in France or abroad, or from public or private research centers.
L'archive ouverte pluridisciplinaire HAL, est destinée au dépôt et à la diffusion de documents scientifiques de niveau recherche, publiés ou non, émanant des établissements d'enseignement et de recherche français ou étrangers, des laboratoires publics ou privés.

\section{(1) (1) $\$$}

Distributed under a Creative Commons Attribution - NonCommercial - NoDerivatives| 4.0 


\section{Norois}

Environnement, aménagement, société

242 | 2017

Espace fluvial, ancrage territorial, travail collaboratif, réseau

\section{Travailler dans un village urbain : pratiques et représentations spatiales des travailleurs créatifs}

Working in an urban village: spatial practices and representations of creative workers

\section{Basile Michel}

\section{OpenEdition}

\section{Journals}

\section{Édition électronique}

URL : http://journals.openedition.org/norois/6042

DOI : $10.4000 /$ norois. 6042

ISBN : 978-2-7535-5895-3

ISSN : 1760-8546

Éditeur

Presses universitaires de Rennes

\section{Édition imprimée}

Date de publication : 22 juin 2017

Pagination : 39-54

ISBN : 978-2-7535-5893-9

ISSN : 0029-182X

\section{Référence électronique}

Basile Michel, «Travailler dans un village urbain : pratiques et représentations spatiales des 


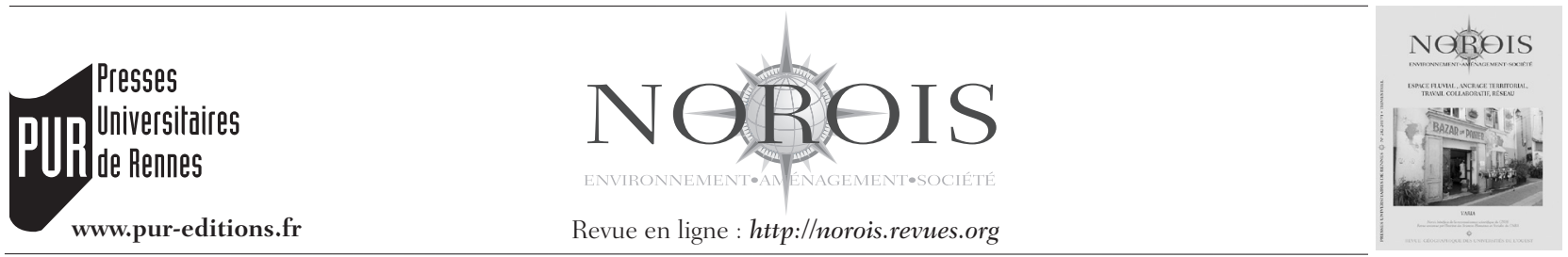

\title{
Travailler dans un village urbain : pratiques et représentations spatiales des travailleurs créatifs
}

\author{
Working in an Urban Village: Spatial Practices and Representations of Creative Workers
}

\author{
Basile MicheL
}

Résumé : L'objectif de cet article est d'analyser les pratiques et les représentations des travailleurs créatifs (artistes, designers...) vis-à-vis de leur quartier d'implantation professionnelle afin de comprendre l'importance qu'ils accordent à cette échelle spatiale. L'analyse s'appuie sur les cas du quartier du Panier (Marseille) et du quartier des Olivettes (Nantes). Une approche méthodologique mobilisant à la fois l'entretien semi-directif, l'analyse de réseaux sociaux et la carte mentale a été mise en place afin d'interroger les travailleurs créatifs, mais aussi les habitants, les chargés de développement du territoire et les acteurs sociaux. Ces enquêtes montrent que le quartier est le support de réseaux collaboratifs de travailleurs créatifs et qu'il est à la fois source et objet de représentations imprégnées de la figure du village pour eux. Elles révèlent que l'ancrage dans le quartier urbain s'articule avec la mobilité des individus et leurs échanges numériques.

\begin{abstract}
This article aims to analyze the practices and representations of creative workers (artists, designers...) regarding their professional neighborhood, in order to understand the importance they accord to this spatial scale. This analysis is based on the examples of the Panier district of Marseille and the Olivettes district of Nantes. I used a methodological approach based on interviews, social network analysis and mental maps to survey not only creative workers but also inhabitants, those responsible for territorial development, and social actors. These investigations reveal that such neighborhoods are places upon which collaborative networks of creative workers develop, and which, to them, are the object of representations influenced by the myth of the village. They indicate that rootedness in the urban neighborhood is combined with individual mobility and digital exchanges.
\end{abstract}

Mots clés : quartier urbain, représentations, réseaux sociaux, cartes mentales

Keywords: urban quarter, representations, social networks, mental maps

\section{INTRODUCTION}

«François et Alice qui bossent chez nous te diraient que l'on est infoutu, incapable, de sortir notre cul du quartier! Vu qu'on a les copains, le boulot et nos logements dans le quartier, je te dis c'est un peu village. »
Ces propos d'un architecte indépendant installé dans le quartier des Olivettes à Nantes révèlent une forme d'ancrage des pratiques professionnelles et personnelles au sein d'un espace réduit. En tant que portion d'espace urbain homogène et clairement identifiable (Lévy et Lussault, 2003), le quartier y 
est valorisé comme un village. Dans le contexte de montée en puissance des pratiques de mobilité, des échanges numériques à distance et d'un habiter poly-topique (Stock, 2006), le discours de cet architecte incite à interroger les liens entretenus par les travailleurs des secteurs culturels et créatifs (art, design...) avec leur quartier d'implantation professionnelle. Les travailleurs créatifs accordent-ils de l'importance à leur quartier professionnel à travers leurs pratiques et leurs représentations?

Ce questionnement se pose avec acuité dans les anciens quartiers populaires et industriels des villes qui concentrent de nombreux travailleurs créatifs (Ambrosino, 2013). L'hypothèse de départ qui a guidé cette recherche est que les travailleurs créatifs développent des relations collaboratives entre eux au sein de ces quartiers, attribuant de l'importance à cet échelon en tant que lieu de travail et d'échange. Identifiée lors des premiers entretiens avec les travailleurs créatifs, la référence au village a amené à la définition d'une seconde hypothèse : les rapports symboliques et pratiques des travailleurs créatifs au quartier relèvent de la figure du quartiervillage enclavé et distinct du reste de la ville. Ces deux hypothèses sont liées par un axe transversal qui interroge la façon dont ces travailleurs articulent l'échelle du quartier avec d'autres espaces physiques (ville...) et immatériels (internet) dans leurs pratiques et leurs représentations. L'ancrage des travailleurs créatifs dans le quartier est-il antagoniste ou complémentaire avec une mobilité des individus à plus large échelle et des échanges numériques?

Afin de mener à bien cette analyse, les quartiers des Olivettes à Nantes et du Panier à Marseille sont mobilisés. C'est notamment la concentration récente de travailleurs créatifs au sein de ces espaces qui les rend pertinents pour cette étude, à l'instar de ce qui a pu être montré concernant le quartier des Olivettes (Petiteau, 2012). Pour comprendre l'importance accordée à ces quartiers par les travailleurs créatifs qui y sont localisés, trois outils méthodologiques ont été mobilisés : l'entretien semi-directif, l'analyse de réseaux sociaux et la carte mentale. Utilisée en complément des entretiens, cette dernière est un outil utile à l'analyse des représentations spatiales des individus (Gieseking, 2013).

Cet article s'articule autour de quatre parties. La première pose les fondements théoriques et métho- dologiques de la recherche. La deuxième servira à présenter les trajectoires évolutives des deux quartiers étudiés. La troisième et la quatrième aborderont successivement les deux hypothèses posées en introduction en analysant d'abord les réseaux collaboratifs locaux puis la prégnance de la figure du village chez les travailleurs créatifs. Pour ces deux aspects, l'articulation entre le quartier et les espaces à plus large échelle sera détaillée.

\section{LE QUARTIER DES TRAVAILLEURS CRÉATIFS COMME CLUSTER ET VIL- LAGE? FONDEMENTS THÉORIQUES ET MÉTHODOLOGIQUES}

\section{Concentration spatiale des travailleurs créatifs : entre opportunité immobilière, espace ressource et retour à la rue}

\section{Les travailleurs créatifs et leur concentration dans les anciens quartiers populaires}

Ayant choisi d'analyser les pratiques et les représentations spatiales des travailleurs créatifs, il est nécessaire de les définir. L'objectif n'est pas de traiter des débats existants autour de l'économie créative (Vivant et Tremblay, 2010), notion dont la pertinence peut être remise en cause (Tremblay, 2008), mais de clarifier ce que sont les « travailleurs créatifs » dans le cadre de cette recherche. Ils sont définis comme les travailleurs des secteurs culturels et créatifs tels que fixés dans le rapport de KEA à la Commission européenne (2006) ${ }^{1}$. Les diverses activités regroupées sous ces secteurs partagent des points communs qui justifient leur regroupement : l'importance de la créativité ${ }^{2}$ des travailleurs (Chantelot, 2010; Liefooghe 2010), le fonctionnement par projets ponctuels (Boltanski et Chiapello, 1999; Menger, 2002), et la forte dimension symbolique et esthétique des productions ( $\mathrm{Scott}$ et Leriche, 2005).

Ces travailleurs se concentrent spatialement dans des quartiers spécifiques des villes (Ambrosino, 2013; Lloyd, 2002). Il s'agit généralement d'es-

\footnotetext{
1. Secteur culturel : arts visuels, arts du spectacle, patrimoine, édition, musique, jeu vidéo, télévision, radio, vidéo et cinéma. Secteur créatif : design, publicité et architecture.

2. La créativité est la capacité cognitive à inventer ou à produire des solutions nouvelles et originales afin de permettre l'innovation ou la résolution de problèmes divers. L'approche multivariée développée en psychologie en définit les principaux déterminants (Lubart, 2003).
} 
paces urbains combinant la proximité du centreville et des prix de l'immobilier abordables (Ovidio et Ponzini, 2014). Les anciens quartiers populaires et/ou industriels répondent à ces critères (Giroud, 2007 et 2011). Ces anciens faubourgs représentent des réserves foncières et immobilières suite à la désindustrialisation des années 1970. Ils possèdent également des qualités sensibles liées à leur histoire sociale, économique et culturelle singulière qui attirent les travailleurs créatifs (Ambrosino, 2013). Leur évolution est souvent influencée par des processus de gentrification consistant en l'augmentation des prix de l'immobilier et le remplacement progressif des populations à bas revenus par des catégories sociales plus aisées (Authier et Bidou-Zachariasen, 2008; Smith, 2003).

\section{Le quartier : un village ressource pour les travailleurs créatifs?}

Différentes recherches montrent que des travailleurs créatifs utilisent leur quartier de résidence comme un espace ressource au niveau professionnel (Collet, 2008 et 2012). Certains sont à la fois habitant et travailleur au sein du même quartier grâce au développement du modèle de l'atelier-logement et du loft (Collet, 2012; Vivant et Charmes, 2008). Les anciens quartiers populaires tels que Belleville (Paris), la Croix-Rousse (Lyon) et South Shoreditch (Londres) sont les lieux privilégiés de ce type de développement. À travers les exemples de Soho et du Bas-Montreuil, Vivant (2009) montre que le quartier peut être un lieu de vie, de vente, d'exposition et de construction identitaire pour les artistes. Accompagnés par une cohorte de travailleurs de secteurs proches des arts (Lloyd, 2002), les artistes participent à créer un réseau relationnel ancré dans le quartier, sorte de cluster culturel localisé (Ambrosino, 2009). Les quartiers investis sont alors le support de réseaux collaboratifs locaux liant les travailleurs créatifs (Mommaas, 2004).

En se concentrant dans d'anciens quartiers populaires, les travailleurs créatifs participent au mouvement de retour à la rue des classes moyennes (Charmes, 2006). Les gentrifiers cherchent dans ces quartiers une forme d'authenticité et une vie de village (Bidou-Zachariasen, 1984 ; Di Méo, 1994). Ils valorisent l'interconnaissance des utilisateurs du quartier et la mixité sociale et culturelle de la popu- lation. La référence au village renvoie à un modèle de société fermée fonctionnant en vase clos partiel où la mobilité des individus est faible (Mendras, 1995; Pelras, 2001). Elle renvoie à la communauté villageoise traditionnelle des $\mathrm{XIX}^{\mathrm{e}}$ et $\mathrm{XX}^{\mathrm{e}}$ siècle. Il importe de saisir si cette référence est partagée par les travailleurs créatifs, et si oui comment s'articulent les échelles du quartier et de la ville dans une société marquée par des modes d'habiter polytopiques où chaque pratique est associée à un lieu spécifique et adapté (Stock, 2006). Cette question de l'articulation du quartier avec les espaces à plus large échelle se pose également au sujet des réseaux collaboratifs tissés par les travailleurs créatifs au sein des quartiers.

\section{Trois méthodes pour une analyse des pratiques et des représentations des travailleurs créatifs}

La méthodologie choisie répond à l'objectif de saisir à la fois les pratiques et les représentations des travailleurs créatifs. Il s'agit donc d'un appareillage méthodologique composite et complémentaire. Trois outils principaux ont été mobilisés : l'entretien individuel semi-directif, l'analyse de réseaux sociaux et la carte mentale (utilisée uniquement en complément des entretiens). Un travail d'observation sur le terrain (vie quotidienne et évènements) et d'étude documentaire (bibliographie, archives...) vient les compléter.

Au total 149 entretiens ont été réalisés entre 2013 et 2016 auprès des travailleurs créatifs (tableau 1) de deux anciens quartiers populaires ${ }^{3}$. Ces derniers ont été choisis pour cette recherche de par la concentration d'activités culturelles et créatives dont ils sont le support. L'échantillonnage des travailleurs créatifs à interroger a été réalisé sur la base des secteurs d'activité représentés au sein de chaque quartier.

Les entretiens avaient pour objectif de comprendre le choix de localisation professionnelle des travailleurs créatifs, leurs parcours de vie, leurs activités professionnelles, leurs réseaux relationnels, leurs pratiques concrètes et leurs représentations du quar-

3. 20 entretiens complémentaires avec d'autres types d'acteurs (habitants, chargés de développement du territoire, acteurs sociaux...) ont été menés afin de saisir la réalité multidimensionnelle de ces espaces. La durée moyenne de ces 169 entretiens $(149+20)$ est d'une heure. L'ensemble de ce travail de terrain est réalisé dans le cadre d'une thèse en géographie. 


\begin{tabular}{|c|c|c|c|c|c|c|}
\hline & \multicolumn{3}{|c|}{ Quartier du Panier } & \multicolumn{3}{|c|}{ Quartier des Olivettes } \\
\hline Sexe & \multicolumn{2}{|c|}{ ㅇ $: 50 \%$} & o: $50 \%$ & \multicolumn{2}{|c|}{ q $: 41 \%$} & 今 : $59 \%$ \\
\hline \multirow{2}{*}{ Répartition par catégorie d'âge } & $20-39$ & $40-59$ & 60 et + & $20-39$ & $40-59$ & 60 et + \\
\hline & $40 \%$ & $39 \%$ & $21 \%$ & $64 \%$ & $32 \%$ & $4 \%$ \\
\hline Secteurs d'activité principaux & \multicolumn{3}{|c|}{$\begin{array}{c}\text { Art : } 64 \% ; \\
\text { Création (vêtements...) : } 17 \%\end{array}$} & \multicolumn{3}{|c|}{$\begin{array}{c}\text { Art : } 25 \% \text {; Architecture : } 17 \% \text {; Design } \\
17 \% \text {; Numérique : } 10 \%\end{array}$} \\
\hline $\begin{array}{l}\text { Nombre moyen de travailleurs } \\
\text { par structure }\end{array}$ & \multicolumn{3}{|c|}{ 1,9 travailleurs } & \multicolumn{3}{|c|}{3,5 travailleurs } \\
\hline \multirow[t]{2}{*}{ Statut dans la structure ${ }^{*}$} & \multicolumn{2}{|c|}{$\begin{array}{l}\text { Chef d'entreprise ou } \\
\text { directeur }\end{array}$} & Salarié & \multicolumn{2}{|c|}{$\begin{array}{l}\text { Chef d'entreprise ou } \\
\text { directeur }\end{array}$} & Salarié \\
\hline & \multicolumn{2}{|c|}{$100 \%$} & $0 \%$ & \multicolumn{2}{|c|}{$92 \%$} & $8 \%$ \\
\hline $\begin{array}{l}\text { Date moyenne d'arrivée } \\
\text { dans le quartier }\end{array}$ & \multicolumn{3}{|c|}{2008} & \multicolumn{3}{|c|}{2010} \\
\hline
\end{tabular}

* La part importante de chefs d'entreprise et de directeurs répond à un choix méthodologique (souhait de s'entretenir avec les dirigeants des structures pour avoir une vision globale de l'activité) mais aussi à un fait lié aux terrains puisque les activités culturelles et créatives sont généralement des microentreprises.

Tableau 1 : Caractéristiques des travailleurs créatifs interrogés en entretien (Source : enquête et réalisation de l'auteur, 2016) Characteristics of creative workers interviewed

tier ${ }^{4}$. L'un des points centraux de l'entretien était de saisir les relations sociales informelles ou formelles et d'ordre personnel ou professionnel tissées à l'échelle du quartier avec d'autres acteurs (travailleurs créatifs, commerçants, habitants...). Pour approfondir ce point, une analyse de réseaux sociaux a été mise en place. L'analyse de réseau mobilisée dans cette étude permet d'identifier si la «proximité géographique» des travailleurs créatifs est activée par une « proximité organisée » (Torre et Rallet, 2005), c'est-à-dire de nature relationnelle. Elle a consisté en l'examen du nombre, de la nature et de l'intensité des relations de chaque travailleur interrogé à l'échelle du quartier. L'objectif était d'identifier dans quelle mesure cet échelon est utilisé par les travailleurs créatifs dans l'exercice de leur métier et comment ils l'articulent avec d'autres échelles spatiales.

Afin d'améliorer la compréhension des représentations des travailleurs créatifs vis-à-vis de leur quartier, la carte mentale a été utilisée. La carte mentale est une " expression cartographique d'une représentation subjective de l'espace » (Lévy et Lussault, 2003, p. 132). Son utilisation en complément des entretiens permet une compréhension fine des représentations spatiales (Moser et Weiss, 2003; Nader, 2012). La passation des cartes mentales a été opérée suivant la «technique de base »(Ramadier, 2003, p. 193).

4. Tous les entretiens ont été analysés suivant une grille thématique transversale reprenant les six axes mentionnés.
Réalisées à la fin des entretiens sur une feuille A4 blanche et vierge, les cartes mentales dessinées par les travailleurs créatifs répondaient à la consigne suivante : "Dessinez votre quartier d'implantation professionnelle en y mettant les éléments importants à vos yeux. » Cette question visait à favoriser l'expression des représentations des personnes interrogées. L'analyse des cartes s'est faite via le repérage de l'échelle spatiale des dessins des travailleurs créatifs (une rue, le quartier, la ville...) et le relevé des éléments représentés. Ces données ont été codées de manière binaire afin de permettre une approche statistique des cartes et des croisements avec les caractéristiques des travailleurs créatifs (secteur, date d'arrivée dans le quartier...). L'objectif de cette analyse est d'identifier si la figure du village enclavé et distinct de la ville est présente dans les représentations spatiales des personnes interrogées.

\section{TRAJECTOIRES DE DEUX ANCIENS QUARTIERS POPULAIRES : LES QUAR- tiers du Panier et des Olivettes}

Les quartiers du Panier à Marseille (figure 1) et des Olivettes à Nantes (figure 2) sont d'anciens quartiers populaires centraux dont l'histoire sociale, économique et culturelle importe dans la compréhension des pratiques et représentations actuelles des travailleurs créatifs. 
Figure 1 : Carte de localisation du quartier du Panier et des activités culturelles et créatives

Location map of the Panier district and of cultural and creative activities

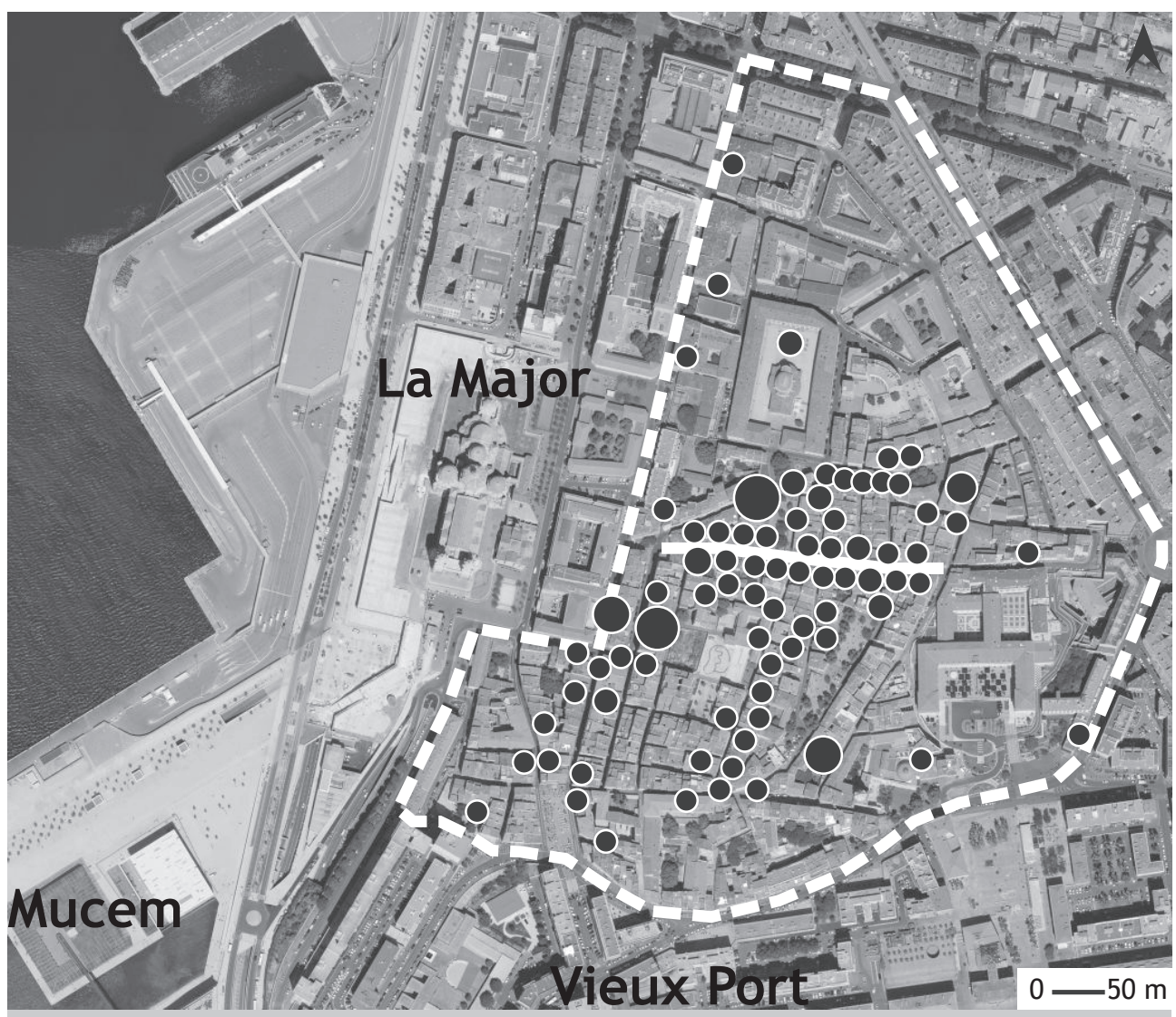

\begin{tabular}{l|l}
\hline Quartier du Panier & Nombre d'activité(s) culturelle(s)
\end{tabular} et créative(s) :

$\begin{array}{ll}1 & \text { Les cercles représentant } \\ 4 & \text { plusieurs activités } \\ 9 & \text { caractérisent des bâtiments } \\ \text { dans lesquels se regroupent } \\ \text { différentes structures. }\end{array}$

Réalisation de l'auteur, 2016. Fond de carte : Géoportail.

\section{Le Panier : continuité populaire d'un ancien village corse}

Le Panier est le principal quartier historique de Marseille. Son implantation sur une butte, ses ruelles piétonnes et escarpées et sa séparation du reste de la ville par des barrières physiques en font un morceau de ville distinct. Il est considéré par ses habitants actuels comme un village (Douart, 2008), faisant référence au mythe du quartier-village isolé dans la ville tel que décrit par Benoit-Guilbot (1986). Cette image est héritée de l'installation massive de corses au cours du XIX ${ }^{\mathrm{e}}$ et de la première moitié du $\mathrm{Xx}^{\mathrm{e}}$ siècle. Ces derniers ont constitué une communauté ancrée dans le quartier et soudée par des liens d'entraide et de solidarité (Attard-Maraninchi, 1997). Depuis cette époque, l'image du village perdure. Les populations immigrées et pauvres qui y trouvent un logement abordable financièrement dans la seconde moitié du $\mathrm{xx}^{\mathrm{e}}$ siècle en assurent la pérennité. Pourtant, du point de vue extérieur, le quartier est un espace répulsif connu pour abriter la mafia. La dégradation des immeubles d'habitation, la pauvreté de ses habitants et la disparition des commerces de proximité accentue cette image négative. Dans les années 1970, les nombreuses opérations de rénovation visant à mettre en valeur sa trame urbaine, son architecture et ses monuments tels que la Vieille Charité ne produiront que peu d'effets à court terme (Benoit-Guilbot, 1986). 


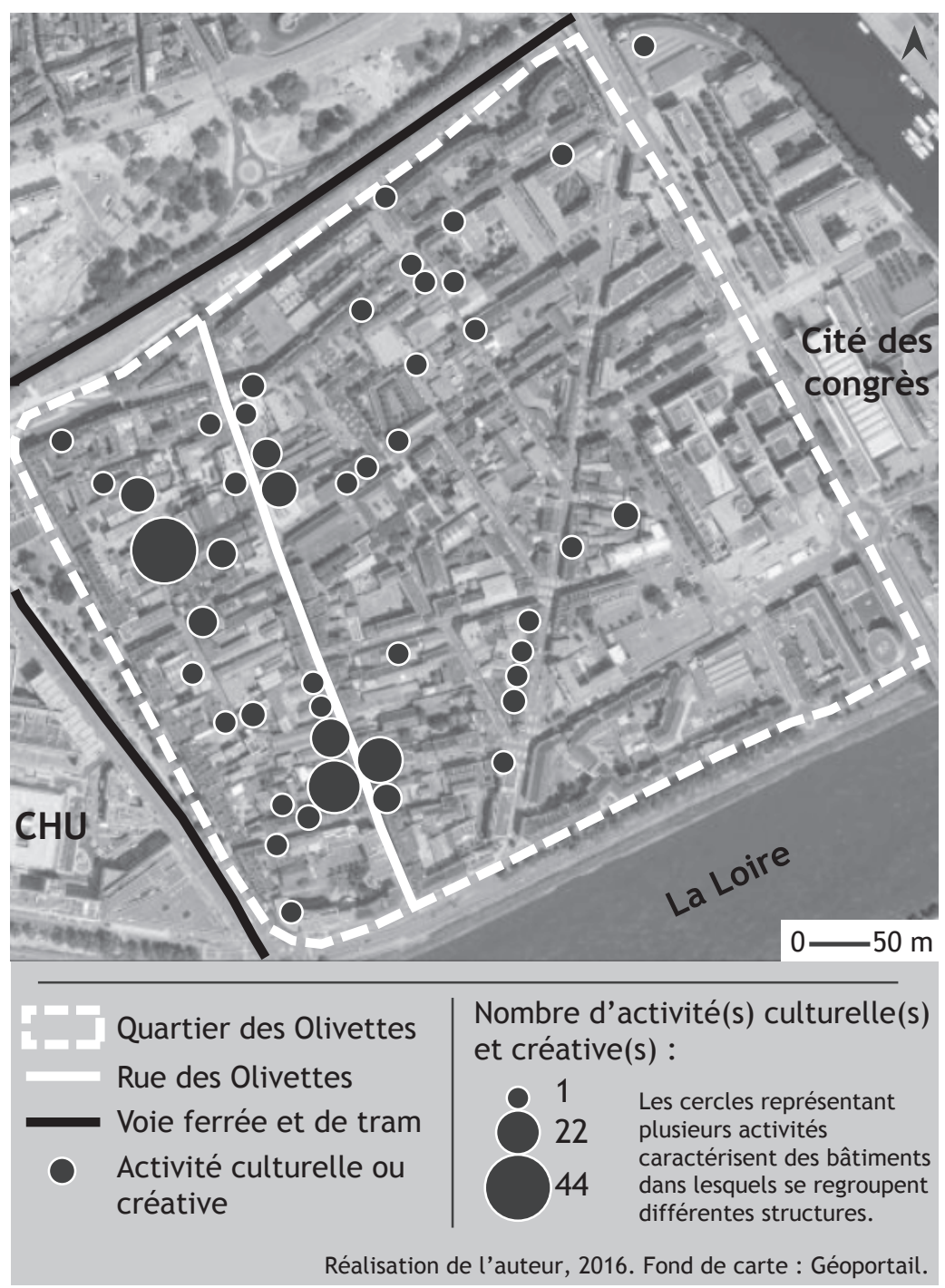

Figure 2: Carte de localisation du quartier des Olivettes et des activités culturelles et créatives Location map of the Olivettes district and of cultural and creative activities
C'est seulement au début des années 2000 que le quartier connait une redynamisation et un changement d'image progressif (Douart, 2008). Ces transformations tiennent à l'implantation d'artisans et d'artistes dans les locaux laissés vacants et aux prix abordables. Ces nouveaux acteurs du quartier donnent au Panier une image positive qui attire notamment les touristes. Le quartier représente aujourd'hui une concentration spatiale d'activités culturelles et créatives (figure 1). 125 activités de ces secteurs ont été recensées en 2016. Elles se répartissent principalement sur les secteurs de l'art (59\%) et de la création (24\%).

Le quartier connait en parallèle une forme de continuité populaire (Giroud, 2007). L'augmentation des prix de l'immobilier entre 1996 et 2006 (Boulay,
2011) n'entraîne qu'une faible croissance des catégories aisées dans la population totale du quartier (Jourdan, 2008). Le Panier continue d'accueillir des populations immigrées précaires financièrement dans des logements indignes (Bouillon, 2003). Les différentes opérations de rénovation n'ont pas suffi à normaliser l'ensemble du parc immobilier qui reste encore aujourd'hui largement marqué par la présence d'immeubles insalubres et en ruine (Iorio, 2011).

\section{Les Olivettes : du faubourg industriel au faubourg créatif}

Officiellement nommé Madeleine-Champ de Mars, le quartier des Olivettes est un ancien fau- 
bourg industriel et populaire. À l'image du Panier, il constitue une enclave urbaine séparée du reste de la ville par des barrières naturelles (la Loire) et des infrastructures (voie ferrée et boulevards). Structurée par de nombreux passages et de nombreuses cours, sa trame urbaine relativement homogène lui confère une unité et une spécificité. Cette trame est héritée du passé industriel et artisanal du quartier. Dès le XVIII ${ }^{\mathrm{e}}$ siècle, des entrepôts, des ateliers et des usines s'implantent dans les cours, les ruelles, les venelles, et les passages du quartier (Wester, 2005). Cette vocation perdure et culmine au cours du Xx $\mathrm{X}^{\mathrm{e}}$ avec le Champ de Mars et les usines $\mathrm{LU}^{5}$ comme symboles. Ces dernières possédaient à la fois une emprise spatiale importante, l'usine occupant jusqu'à $40000 \mathrm{~m}^{2}$ en 1913 , et une place économique stratégique avec 1200 employés dans les années 1960 (Couvrand, 2005). Le quartier est à cette époque le lieu d'une vie ouvrière emblématique des faubourgs industriels.

Le déclin industriel amorcé dans les années 19601970 plonge le quartier des Olivettes dans une spirale négative de dégradation urbaine. La disparition de l'activité industrielle provoque le développement de friches dont l'image négative imprègne l'ensemble du quartier alors reconnu par les nantais comme un « coupe gorge». Le lancement de la Zone d'Aménagement Concerté (ZAC) en 1989 marque un tournant. La municipalité met en place un projet de rénovation progressif visant à faciliter l'implantation d'activités des secteurs culturels et créatifs (Petiteau, 2012). Ces activités sont ciblées car elles ont «la capacité de poursuivre l'histoire faubourienne » du quartier (Chargé d'opérations à Nantes Métropole Aménagement, entretien no 96, juillet 2015). Elles profitent de prix de l'immobilier accessibles dans un espace central de la ville. L'occupation éphémère des bâtiments dégradés en attente de réhabilitation par des artistes est encouragée pour redonner vie au quartier (Roy, 2004). À mesure que la rénovation progresse, son image se transforme. Les travailleurs créatifs y jouent un rôle moteur et le quartier s'affirme au début des années 2000 comme le quartier de la création officieux de la ville. Alors que la ville de Nantes a établi un Quartier de la Création officiel sur lîle de Nantes, les travail-

5. Il s'agit de l'ancienne biscuiterie LU dont l'une des tours symboliques du quartier a été préservée. Depuis 2000, une partie des bâtiments est reconvertie en centre d'art hybride, le Lieu Unique. leurs créatifs implantés aux Olivettes revendiquent leur appartenance au «vrai » quartier de la création de la ville : "Pour moi le quartier de la création il est là, il n'est pas sur l'île de Nantes. " (Designer du quartier des Olivettes, entretien n ${ }^{\circ} 78$, juillet 2015.) Cette revendication s'appuie sur l'existence actuelle d'une concentration d'activités culturelles et créatives au sein de ce territoire (figure 2). Ces activités représentent 249 structures appartenant à des secteurs plus diversifiés qu'au Panier (architecture: $19 \%$, art : $17 \%$, design : $17 \%$, numérique : $11 \%$ ).

\section{UN CLUSTER DE TRAVAILLEURS CRÉATIFS AU SEIN DES QUARTIERS : ENTRE ANCRAGE LOCAL ET ARTICULA- TION DES ÉCHELLES SPATIALES}

\section{Réseaux collaboratifs de travailleurs créatifs : un ancrage dans le quartier}

À l'intérieur des quartiers du Panier et des Olivettes se constituent des réseaux collaboratifs liant les travailleurs créatifs entre eux. Ils entretiennent (1) des relations marchandes telles que la réalisation de projets collectifs et de prestations de service et (2) non marchandes comme des prêts de matériel, de la mutualisation de moyens, de l'entraide, des échanges d'information et de la mise en relation ${ }^{6}$. À l'image du quartier du Bas-Montreuil dans lequel se constitue « une sorte de marché du travail secondaire »(Collet, 2008, p. 136), les quartiers du Panier et des Olivettes sont animés d'une dynamique socioéconomique propre à leur périmètre. Au sein du Panier, les travailleurs créatifs tissent en moyenne quatre relations avec d'autres travailleurs des secteurs culturels et créatifs implantés dans le quartier. Pour les Olivettes, ce même chiffre s'élève à onze ${ }^{7}$. Dans les deux quartiers, les travailleurs créatifs isolés sont rares $\left(15 \%^{8}\right)$. Ces relations varient en termes d'intensité entre des échanges quotidiens et des échanges ponctuels (quelques fois par an) en passant par une catégorie

6. Cette typologie est notamment utilisée par Emin et Sagot-Duvauroux (2016).

7. Tous les travailleurs créatifs du Panier et des Olivettes n'ayant pu être rencontrés, ces chiffres sous-estiment le nombre réel de relations entretenues à l'échelle du quartier. Les travailleurs rencontrés en entretien ont en moyenne respectivement 6 et 15 relations au Panier et aux Olivettes.

8. Pour la même raison, ce chiffre surestime le nombre de travailleurs isolés. Ainsi, seulement $4 \%$ des travailleurs interrogés en entretien n'ont pas de relation au sein de leur quartier. 
intermédiaire (échanges réguliers). Au Panier, les relations sont principalement ponctuelles (48\%) alors qu'aux Olivettes elles ont une intensité plus élevée (69\% pour la catégorie intermédiaire). La nature des relations entretenues par les travailleurs créatifs varie également entre le Panier et les Olivettes.

Le premier est un lieu de création mais la proximité géographique des travailleurs créatifs est principalement activée pour la partie diffusion de la chaîne de valeur. Leurs collaborations correspondent par exemple à la création d'événements collectifs visant à attirer du public. Le festival La Nuit de l'Instant est une illustration des productions collectives réalisées par le réseau collaboratif local. Ce festival propose une déambulation dans le Panier autour de la thématique de l'image. Il est organisé par une association artistique du quartier qui associe une dizaine d'autres travailleurs et lieux créatifs. L'importance des coopérations portées sur la diffusion s'explique à la fois par les profils des professionnels présents (nombreux artistes et créateurs ayant des ateliers-galeries) et la dimension touristique du lieu. Ainsi, le réseau créatif du Panier est tourné vers la diffusion et la vente, alors que celui des Olivettes est centré sur la création. Si ce quartier peut être un espace de diffusion, l'agglomération spatiale des travailleurs créatifs résulte de leur volonté de collaborer dans la réalisation de projets et de prestations de services. Ces coopérations prennent des formes variées allant du partage de techniques de création entre deux artistes à la réalisation d'un site internet d'un architecte par un web designer. Les travailleurs créatifs des Olivettes répondent aussi de manière collective à des demandes de clients en associant leurs compétences pour proposer une réponse globale. Des équipes de travailleurs indépendants se mettent ainsi en place au sein du quartier. Les appels d'offres dans le domaine de la construction font par exemple l'objet de réponses collectives entre plusieurs agences d'architecture et des paysagistes implantés aux Olivettes.

Dans les deux cas, les réseaux professionnels ancrés dans les quartiers donnent de l'importance à cet échelon spatial. Les travailleurs créatifs y trouvent des collaborateurs, des compétences complémentaires, des prestataires, des commanditaires, des professionnels avec qui discuter et même des amis. Au-delà des aspects marchands et formels, les relations sociales informelles sont déterminantes au sein du réseau collaboratif. Un système d'entraide est mis en place et lie les travailleurs créatifs les uns aux autres: "Tu as une forme de "je te reconnais parce que tu m'as filé un coup de main". " (Gestionnaire d'une association implantée aux Olivettes, entretien $n^{\circ} 72$, juillet 2015.) Le développement de leurs activités s'en trouve stimulé et leur bien-être au travail amélioré. Le quartier s'affirme alors comme une combinaison de ressources et un élément du capital spatial (Lévy et Lussault, 2003) des travailleurs créatifs.

\section{Articulation des échelles par les travailleurs créatifs : entre ancrage dans le quartier et ouverture}

Ces réseaux ancrés dans les quartiers du Panier et des Olivettes ne constituent pas au niveau économique des systèmes indépendants fonctionnant en vase clos. Les travailleurs créatifs s'appuient sur les relations locales d'entraide et de coopération pour développer leur activité mais ils ne s'y limitent pas. Leurs publics, leurs clients et leurs partenaires se situent à des échelles spatiales plus larges. Trois éléments en attestent.

Premièrement, tous les travailleurs créatifs rencontrés possèdent et utilisent un site internet pour assurer leur visibilité ${ }^{9}$. Cette présence sur internet témoigne de la volonté des travailleurs créatifs de développer leur activité à destination de publics et de clients d'horizons géographiques divers. Cela se traduit dans la fréquentation des théâtres du Panier et des Olivettes dont les publics sont majoritairement extérieurs aux quartiers. Les créateurs d'objets n'hésitent pas à aller plus loin dans l'utilisation de leur site en proposant des services de vente en ligne.

Deuxièmement, les artistes et les créateurs participent à des salons d'art et exposent leurs œuvres dans des lieux (galeries d'art, magasins de créateurs) extérieurs au quartier. Leur objectif est de se rendre visible et de gagner en notoriété. Dans le cas du Panier, $74 \%$ des artistes et des créateurs utilisent ces procédés en plus de leur propre atelier-galerie.

Troisièmement, la proximité de la gare SNCF est valorisée par les travailleurs créatifs comme un atout. Cela est particulièrement le cas aux Olivettes

9. Six exceptions ont toutefois été répertoriées. 
où $21 \%$ des personnes interrogées considèrent cet élément comme une caractéristique importante du quartier qui facilite la venue de leurs clients et leurs propres déplacements professionnels.

L'ancrage dans le quartier est complémentaire du développement des activités des travailleurs créatifs dans des espaces physiques (ville, pays...) et immatériels (internet) plus larges. Le quartier apparaît comme un lieu d'entraide et de coopération que les travailleurs créatifs articulent avec des échelles spatiales d'action qui dépassent le cadre local. Le directeur d'une agence de communication du quartier des Olivettes témoigne ainsi de l'importance de l'entraide et du prêt de matériel à l'échelle du quartier, mais de l'ouverture économique à une plus grande échelle (nationale principalement) : «Dans l'entraide, dans le fait de se filer un coup de main parce qu'on a un super copieur couleur et que tout le monde n'en a pas dans la rue et que parfois ça peut dépanner, ça semble juste évident en fait. [...] Mais après dans le business en tant que tel, il n'est pas lié en fait, il n'est pas développé à l'échelle du territoire, de la rue ou du quartier, il est développé de manière beaucoup plus large que ça. » (Entretien $\mathrm{n}^{\circ}$ 1, avril 2013.) Le cas de quatre travailleurs indépendants implantés dans le quartier des Olivettes est une illustration de cette articulation entre le réseau collaboratif du quartier et l'échelle globale. Ces travailleurs sont graphistes et illustrateurs. Sans se connaître au préalable, ils se sont installés entre 2012 et 2014 dans un même espace de travail partagé qui regroupe une quinzaine de travailleurs créatifs. Ils ont fait le choix de quitter Paris pour venir travailler à Nantes afin de se rapprocher de leurs familles et de se soustraire aux pressions inhérentes à la capitale française. Malgré ce déménagement, trois d'entre eux ont conservé leurs clients parisiens et continuent d'échanger avec eux à distance. Ils profitent également de la proximité de la gare pour se rendre de manière ponctuelle et temporaire dans leurs locaux. Parallèlement, l'installation dans le quartier des Olivettes a permis aux quatre indépendants de s'intégrer dans un réseau complémentaire à l'échelle locale. Chacun d'entre eux possède aujourd'hui au sein du quartier entre quatorze et dix-sept relations dont $13 \%$ sont marchandes (prestations de services) et $87 \%$ exclusivement non marchandes (entraide, échanges d'information...).
Au final, les quartiers du Panier et des Olivettes sont le lieu de clusters créatifs localisés qui s'articulent avec des échelles spatiales plus larges.

\section{LA FIGURE DU VILLAGE : LE QUARTIER DES TRAVAILLEURS CRÉATIFS COMME ENCLAVE URBAINE}

Les réseaux collaboratifs des quartiers du Panier et des Olivettes mettent en relation les travailleurs créatifs. Cette mise en réseau participe à l'émergence de représentations empreintes de la figure du quartier-village marquée par l'interconnaissance des utilisateurs de ce territoire.

\section{Le monde symbolique et spatial du quartier-village}

Pour les travailleurs créatifs, les quartiers du Panier et des Olivettes s'apparentent à un village caractérisé par sa mixité sociale et culturelle. Les représentations des travailleurs créatifs sont empreintes du mythe du village symbolisé par la formule consacrée : "C'est un village, tout le monde se connaît. » (Brocanteur-recycleur d'objet installé au Panier, entretien $n^{\circ} 134$, novembre 2015.) Les motsclés ${ }^{10}$ utilisés par les travailleurs créatifs pour définir leur quartier témoignent de l'importance de cette représentation. Les mots "village », " quartier», « faubourg », «populaire », « convivial », « mixité » et « vie sociale » font partie des plus cités. Chaque mot utilisé a été classé suivant le monde symbolique auquel il se réfère. Les figures 3 et 4 présentent les différents mondes symboliques dans lesquels s'insèrent les mots-clés utilisés par les travailleurs créatifs des deux quartiers.

Leur analyse lexicale révèle que les représentations des travailleurs créatifs sont dominées par des aspects ayant trait à la figure du village. Dans le cas du Panier, c'est la configuration urbaine du village corse perché sur sa butte qui prédomine (49 occurrences). Cette dimension se double d'une mixité sociale et culturelle de la population soudée par un dynamisme social et solidaire (34 occurrences). Dans le cas des Olivettes, le monde du village est mobilisé pour mettre en valeur la configuration du faubourg enclavé constitué d'une trame urbaine pré-

10. À la fin des entretiens avec les travailleurs créatifs, des mots-clés définissant pour eux leur quartier professionnel étaient demandés. 


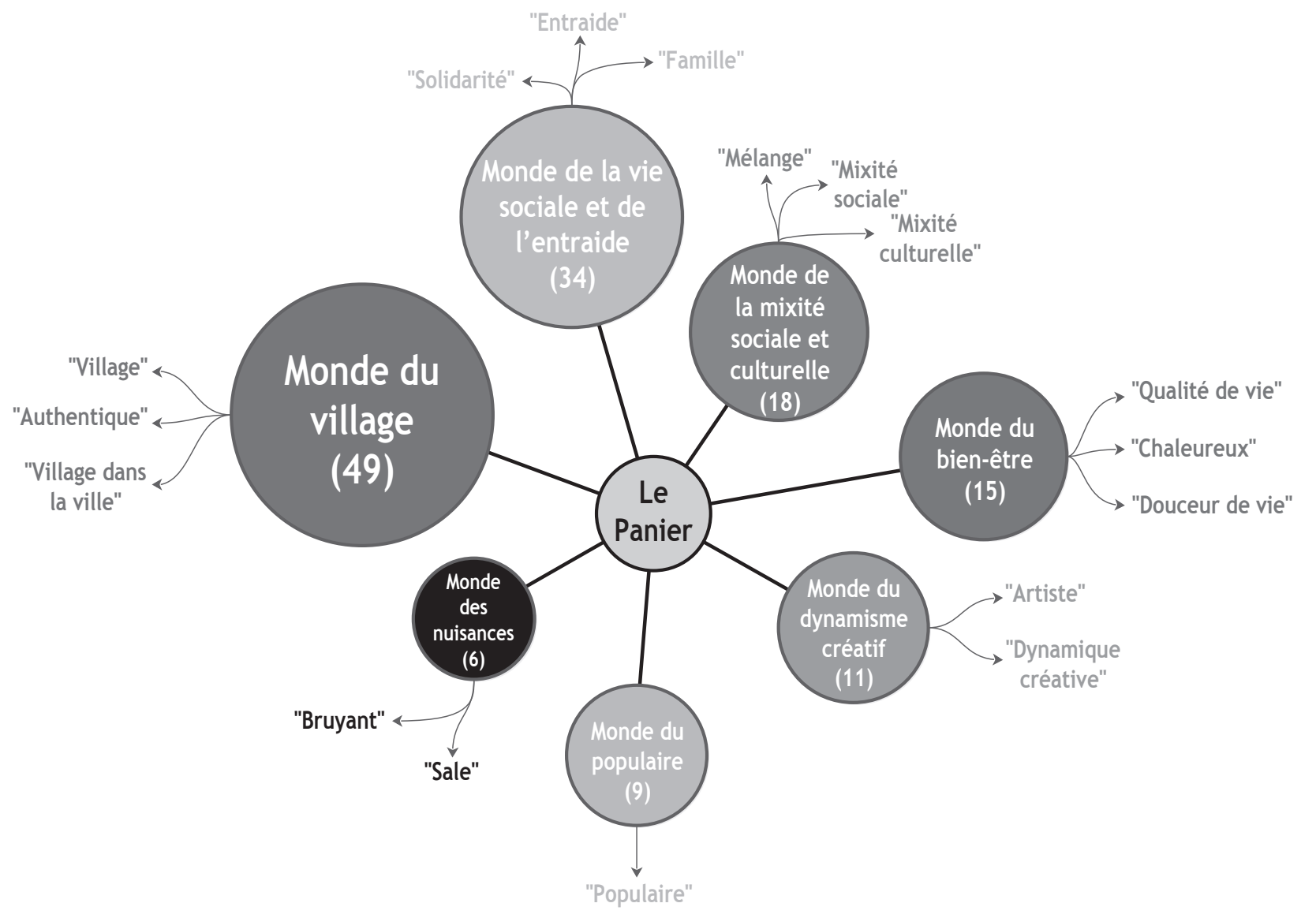

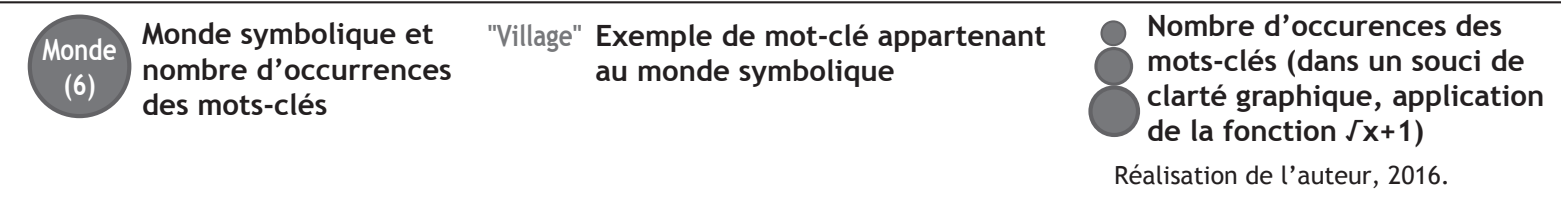

Figure 3 : Mondes symboliques des mots-clés utilisés par les travailleurs créatifs pour définir le quartier du Panier Symbolic worlds of the keywords used by creative workers to define the Panier district

servant les traces mémorielles du passé industriel et ouvrier du quartier (51 occurrences). Ce monde est lié à celui de la mixité sociale et fonctionnelle (23 occurrences). Les créatifs valorisent la continuité populaire au sein du quartier. Ils sont attentifs à l'évolution sociale et urbaine de ce territoire comme en témoigne leur lecture de l'ouvrage de Petiteau (2012) qu'ils me recommandent régulièrement ${ }^{11}$. Ils valorisent aussi le maintien d'une vie sociale habitante en parallèle du développement d'activités professionnelles. La présence d'habitants apparaît comme un élément important de

11. Cet ouvrage présente l'évolution du quartier des Olivettes dans le cadre de la ZAC Madeleine-Champs de Mars au travers de témoignages des différents acteurs du territoire (élus, habitants, artistes...). leur rapport positif au quartier. Enfin, les mondes du bien-être et du dynamisme collaboratif font référence aux nombreux échanges interpersonnels qui se tissent ou peuvent se tisser au sein du «village » des Olivettes dans une ambiance qualifiée de « chaleureuse ».

Les cartes mentales expriment des représentations qui coïncident avec le discours du village dans la ville en présentant les quartiers comme des enclaves urbaines (figure 5). Les frontières des deux quartiers sont les éléments les plus représentés derrière la rue du Panier et la rue des Olivettes. Les trois limites du triangle du Panier sont présentes dans $47 \%$ des cartes. Pour le quartier des Olivettes, les contours ouest $(100 \%)$, est $(38 \%)$, nord $(77 \%)$ et sud $(79 \%)$ 


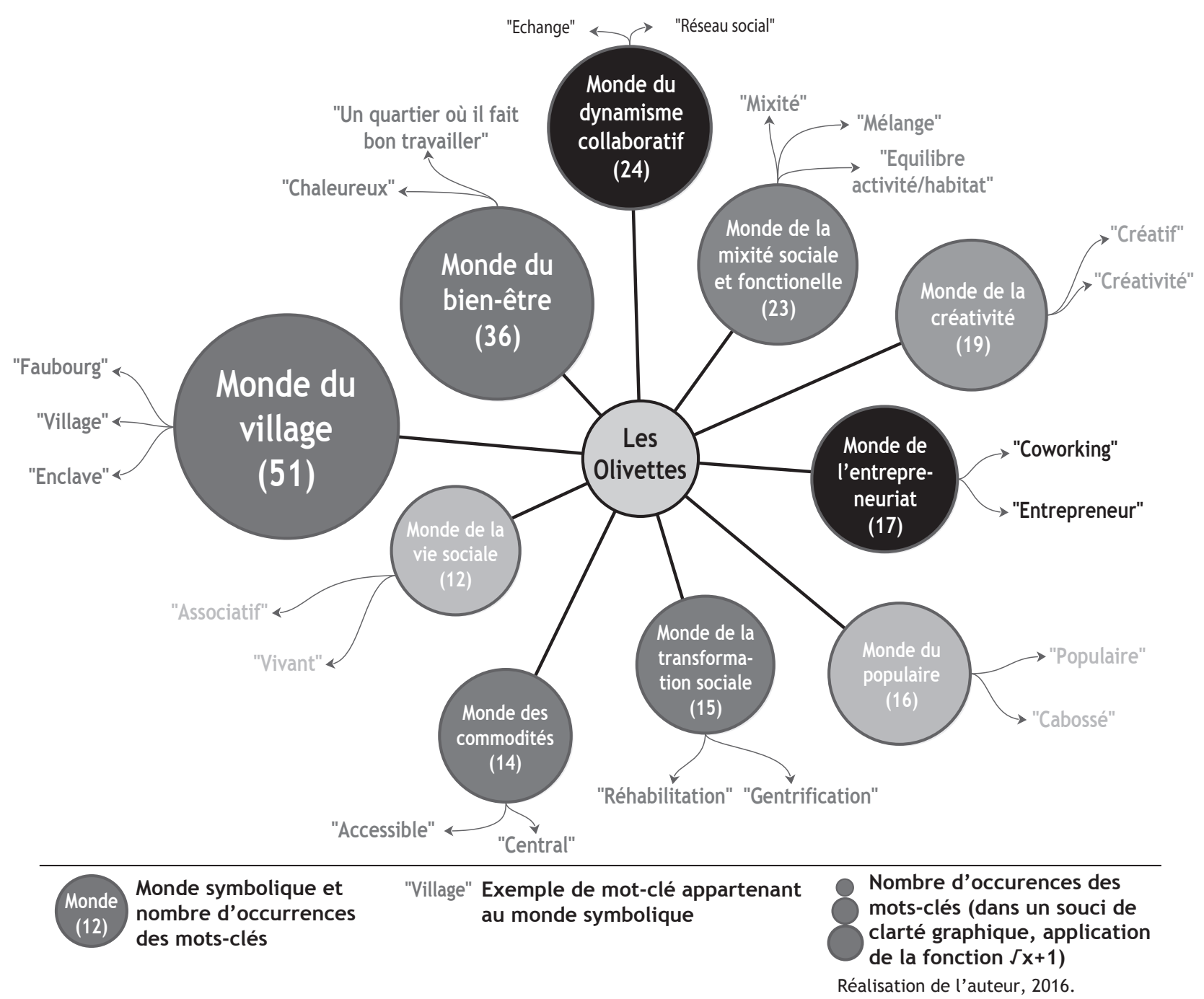

Figure 4 : Mondes symboliques des mots-clés utilisés par les travailleurs créatifs pour définir le quartier des Olivettes Symbolic worlds of the keywords used by creative workers to define the Olivettes district

sont également fortement présents. Ils prennent la forme des barrières réelles existantes autour du quartier (figure 2). L'importance des limites du quartier marque l'existence d'une entité bien délimitée distincte du reste de la ville dans les représentations. On sort des Olivettes et on y entre, on monte au Panier et on descend à Marseille : «Au-dessus il y a Jean-Jean qui a 75 ans et au début il me disait "Oh Phil je vais à Marseille tu veux quelque chose?" Alors j'éclatais de rire mais maintenant je dis pareil quoi. On descend à Marseille, ici on n'est pas à Marseille. " (Créateur installé au Panier, entretien no 104, octobre 2015.)

La distinction avec les villes de Marseille et de Nantes est également marquée par la faible représentation d'éléments de contexte des quartiers sur les cartes mentales. Le centre-ville n'est ainsi dessiné que très rarement $(10 \%)$. Cet isolement du quartier présent dans les représentations des travailleurs créatifs est particulièrement visible sur la carte mentale d'une créatrice du Panier (figure 5). Le Panier y est dessiné comme un triangle composé de la rue du Panier, de son atelier, de son logement et de ses nombreux amis présents dans le quartier (représentés par les croix). Ce périmètre fermé est plaqué sur la ville de Marseille qui constitue un environnement urbain flou qualifié d'« inconnu » et de « danger». Cette carte symbolise une forme d'enfermement et d'isolement du Panier vis-à-vis de l'extérieur. Elle illustre aussi l'image d'un quartiervillage composé de personnes connues. 


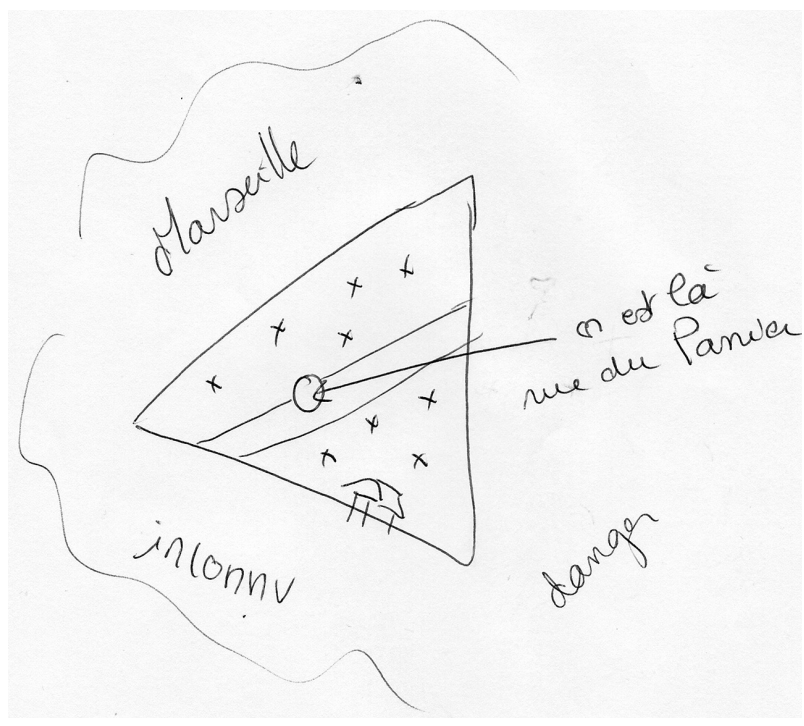

Figure 5 : Carte mentale d'une créatrice d'objets du quartier du Panier Mental map of the Panier district by a local creator of decorative objects

Les représentations d'un quartier-village mixte socialement sont réaffirmées face aux transformations que connaissent les deux quartiers. Malgré la rénovation progressive du bâti et l'arrivée de nouvelles populations de travailleurs et d'habitants plus aisées, les travailleurs créatifs conservent l'image du village mixte dans leurs représentations : «Ici c'est vraiment un beau mélange, tout le monde dit que ça devient un quartier bobo, ce qui n'est pas vrai. C'est un quartier qui reste très populaire. » (Créatrice installée rue du Panier, entretien $n^{\circ} 100$, octobre 2015.) Cette représentation correspond à la réalité puisque l'installation de populations aux revenus plus élevés, dont les travailleurs créatifs font partie, s'accompagne pour le moment d'un maintien des populations les plus pauvres, tant au Panier qu'aux Olivettes ${ }^{12}$.

\section{Sortir du village : articulation des échelles du quartier et de la ville par les pratiques des travailleurs créatifs}

La valorisation du quartier comme village est toutefois nuancée par des représentations mettant en

12. Les chiffres de l'Insee attestent d'une médiane des revenus annuels par Unité de Consommation (UC) en 2011 inférieure aux villes de Nantes et de Marseille. De plus, le premier décile des revenus annuels par UC à la même date est très bas $(5.263 €$ pour les Olivettes et $285 €$ pour le Panier). Un processus de gentrification est tout de même en cours et pourrait faire évoluer ces constats. Le dernier décile des revenus annuels par UC est ainsi passé entre 2001 et 2011 de 25.937 à $36.429 €$ aux Olivettes et de 20.198 à $27.677 €$ au Panier. avant le contrôle social et l'absence d'intimité qui en découle. Ce constat est particulièrement vrai pour le Panier. Les rues y ont des yeux (Jacobs, 1991): «Là on parle, dehors on entend ce que l'on dit, donc il n'y a pas d'intimité, jamais. Ça c'est important de le savoir. » (Créateur installé au Panier, entretien $\mathrm{n}^{\circ}$ 104, octobre 2015.) Ces représentations participent à la construction de certaines pratiques spatiales des travailleurs créatifs que l'on peut séparer en deux catégories.

La première concerne les travailleurs qui sont également des habitants des quartiers du Panier et des Olivettes. Ils représentent respectivement $51 \%$ et $12 \%$ des personnes interrogées. Cette localisation résulte d'une volonté de proximité des lieux de travail et de résidence permettant la circulation piétonne. C'est dans l'extrême majorité des cas un véritable choix de vie. Pour ces travailleurs, la concentration des lieux de travail, de résidence et souvent de loisirs au sein du même périmètre géographique entraîne un fort ancrage de leurs pratiques quotidiennes. Ils ressentent alors le besoin de sortir régulièrement de la bulle que représente le quartier. Ces travailleurs créatifs profitent de l'intégration du Panier et des Olivettes dans les villes de Marseille et de Nantes pour assouvir un besoin de respiration. Cela se traduit par des pratiques diverses hors du quartier à une fréquence hebdomadaire (balades, courses...). Les échelles spatiales du quartier et de la ville s'articulent alors: "On a les deux aspects dans ce quartier. On a le côté village, où tout le monde se connaît, où ça papote, on se soucie de tout le monde, mais en même temps on est dans une très grande ville donc à partir du moment où on veut être un peu discret, c'est aussi possible. [...] Il y a un anonymat qui redevient quand même possible vu la taille de la ville. Moi j'ai besoin de ces deux aspects pour être bien. » (Artiste installée au Panier, entretien n ${ }^{0} 123$, novembre 2015.)

La seconde catégorie a trait aux travailleurs n'habitant pas dans les quartiers du Panier et des Olivettes (49\% et $88 \%$ des personnes interrogées). Ces travailleurs habitent majoritairement à Marseille (91\%) ou à Nantes (54\%), tandis que les autres résident dans des espaces périurbains de ces deux villes ( $9 \%$ et $46 \%$ ). De par leur localisation résidentielle, ces travailleurs sont amenés à pratiquer quotidiennement des espaces à l'échelle de la ville et de la métropole. Les lieux de résidence et de travail sont distincts et chacun est dédié à une fonction 
singulière. Ces pratiques multi scalaires s'inscrivent dans une logique d'habiter poly-topique (Stock, 2006). Dans le cas du Panier, cette disjonction des espaces du logement et du travail relève d'un choix (inverse à celui fait par les travailleurs-habitants). Leur volonté est de ne pas s'enfermer dans un espace géographique et social fermé, à l'image d'une artiste s'étant posée la question d'habiter au Panier avant de renoncer : "J'aime beaucoup le Panier, mais en même temps être complètement au Panier, je ne suis pas sûr que j'aimerais parce que quand même ça reste un endroit fermé, c'est petit, c'est fermé et à cinq mètres du Panier ce n'est plus le Panier, on sent bien, [...] on est dans cet univers un peu clos. Je pense que j'ai ma dose comme ça [en y travaillant sans y habiter]. J'aime bien mais j'ai ma dose. » (Entretien $\mathrm{n}^{\circ} 188$, mai 2016.)

Cette articulation entre le quartier et la ville dans les représentations et les pratiques des travailleurs créatifs amène à considérer la référence au village comme excessive vis-à-vis de la réalité. Le quartier n'est qu'un espace parmi d'autres dans leurs pratiques quotidiennes au contraire du fonctionnement des anciennes sociétés villageoises (Le Goff, 2012; Mendras, 1995). De plus, par la valorisation de la figure du village et de ses dimensions historiques et sociales (Garden et al., 1986), les travailleurs créatifs justifient leur choix de localisation et leur mode de vie à l'image du retour à la rue des classes moyennes dans les quartiers populaires de centreville (Charmes, 2005). La mise en valeur de la ville traditionnelle telle qu'entendue par Jacobs (1991) rend valorisable socialement et psychologiquement le fait de travailler dans un quartier choisi en partie pour ses prix de l'immobilier abordables.

\section{Différenciation des représentations des travailleurs créatifs : nuances de la figure du village}

La figure du village est largement partagée dans les représentations des travailleurs créatifs des quartiers du Panier et des Olivettes. L'analyse des cartes mentales amène toutefois à nuancer le partage homogène de cette figure. Elle fait apparaître une différenciation majeure entre les travailleurs créatifs représentant leur quartier à l'échelle d'une rue ou d'un espace plus réduit (35\% au Panier et $45 \%$ aux Olivettes) et ceux le représentant à l'échelle du quartier telle qu'identifiée précédemment $(65 \%$ au Panier et $55 \%$ aux Olivettes) ${ }^{13}$ (figures 1 et 2 ). Ainsi, la figure du village enclavé n'est pas partagée par tous les travailleurs créatifs. Au contraire du second, le premier type de représentations n'y correspond pas. Le quartier n'est pas représenté comme une enclave dans la ville, sorte de microsociété isolée du reste du tissu urbain. Il se réduit à des lieux situés autour du bureau du travailleur créatif (figure 6).

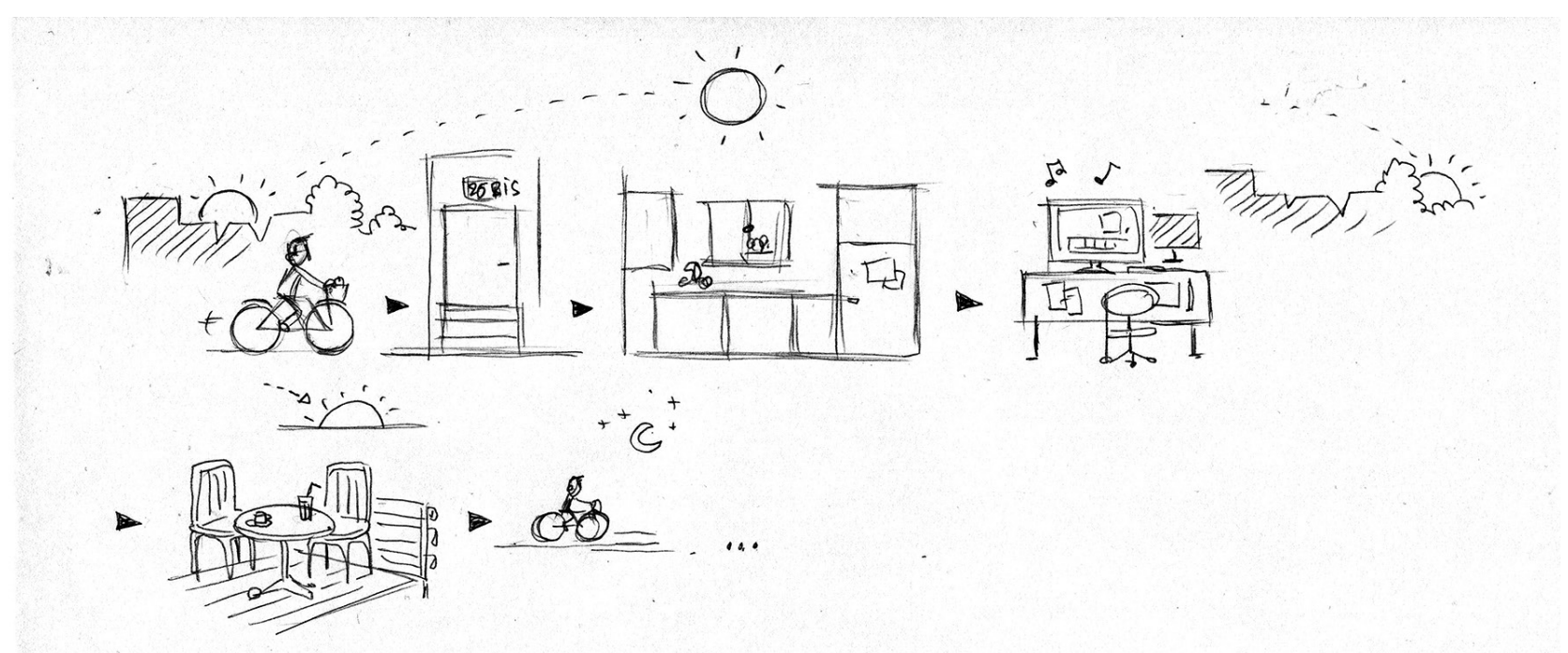

Figure 6 : Carte mentale d'une story-boarder et illustratrice du quartier des Olivettes

Mental map of the Olivettes district by a local storyboarder
13. Les représentations imagées du quartier ont été exclues volontairement pour cette partie de l'analyse car elles ne présentent pas de dimension spatiale. Elles représentent $13 \%$ des cartes au Panier et aux Olivettes. 
L'ancienneté dans le quartier et le secteur d'activité apparaissent comme les facteurs explicatifs de cette distinction entre les deux types de représentations. Les travailleurs les plus anciennement installés connaissent plus en profondeur les lieux, les limites et les acteurs du quartier. Ils en ont une représentation plus complète. Leur échelle de représentation s'en trouve agrandie (tableau 2).

Le secteur d'activité influence également le rapport symbolique entretenu par les travailleurs créatifs avec le quartier. Au Panier, les artistes ont une représentation à l'échelle du quartier conforme à la figure du village enclavé ( $80 \%$ des cartes). Cette représentation est moins largement partagée au sein des autres secteurs d'activité (33\%). Dans le cas des Olivettes, les designers et les acteurs du numérique sont davantage centrés sur la rue des Olivettes (75\% et $50 \%$ ), au contraire des artistes $(25 \%)$. Ces derniers sont par la nature de leur activité incités à aller vers les utilisateurs du quartier et à se saisir des différents lieux qui le composent. L'organisation d'évènements culturels au sein du quartier leur permet de découvrir en profondeur le territoire proche. Ils en utilisent les formes pour créer de l'art urbain. Ils acquièrent une connaissance et une représentation du quartier plus complète. À l'inverse, les designers et les acteurs du numérique demeurent centrés sur leur activité professionnelle qui se cantonne principalement à leur bureau et à ceux de leurs partenaires. Cela se retranscrit dans leurs représentations du quartier à une échelle spatiale réduite telle qu'une rue (figure 7) ou un bureau (figure 6). Le reste du quartier est perçu comme une entité extérieure à leur espace professionnel.

Les représentations à des échelles restreintes illustrent un plus faible intérêt pour le quartier tel que défini classiquement. Elles montrent une restriction spatiale des lieux considérés comme importants dans l'espace professionnel de proximité. Elles nuancent l'homogénéité des représentations des travailleurs créatifs suivant la figure du quartier-village. Cette figure demeure toutefois une référence collective, en particulier chez les travailleurs créatifs installés depuis plusieurs années au sein du Panier et des Olivettes.

\section{Conclusion}

Les travailleurs créatifs entretiennent une pluralité de rapports pratiques et symboliques avec leur quartier d'implantation professionnelle. Cet espace représente une ressource grâce au réseau collaboratif qui s'y ancre. Il fait l'objet de représentations majoritairement imprégnées de la figure du village dans la ville. Les exemples du Panier et des Olivettes montrent l'importance que revêt encore aujourd'hui cette échelle de proximité du quartier à la fois en tant que support et agent de pratiques et de représentations. À l'heure des réseaux sociaux numériques et de l'habiter poly-topique, les réseaux collaboratifs locaux mis en place par les travailleurs créatifs témoignent de la persistance du quartier comme échelle importante dans le déroulement d'activités professionnelles. Le quartier n'est toutefois qu'un espace physique et relationnel parmi d'autres pour les travailleurs créatifs. Les réseaux collaboratifs locaux et les représentations du village enclavé s'articulent avec les échelles spatiales plus larges via les pratiques des travailleurs créatifs.

Au travers de cet article il apparaît que la prise en compte des pratiques et des représentations des individus, y compris dans un contexte professionnel,

\begin{tabular}{|c|c|c|}
\hline & Quartier du Panier & $\begin{array}{c}\text { Quartier } \\
\text { des Olivettes }\end{array}$ \\
\hline Part des cartes à l'échelle du quartier pour les travailleurs créatifs arrivés avant 2010 & $82 \%$ & $83 \%$ \\
\hline $\begin{array}{l}\text { Part des cartes à l'échelle du quartier pour les travailleurs créatifs arrivés à partir de } \\
2010\end{array}$ & $53 \%$ & $45 \%$ \\
\hline $\begin{array}{l}\text { Date moyenne d'arrivée dans le quartier des travailleurs créatifs dont la carte est à } \\
\text { l'échelle du quartier }\end{array}$ & 2007 & 2008 \\
\hline $\begin{array}{l}\text { Date moyenne d'arrivée dans le quartier des travailleurs créatifs dont la carte est à } \\
\text { l'échelle de la rue ou d'un espace plus réduit }\end{array}$ & 2010 & 2012 \\
\hline
\end{tabular}

Tableau 2 : Influence de l'ancienneté dans le quartier dans l'échelle de représentation des cartes mentales des travailleurs créatifs (Source : enquête et réalisation de l'auteur, 2016.) Influence of length of presence in the neighborhood on the scale of representation of creative workers' mental maps 


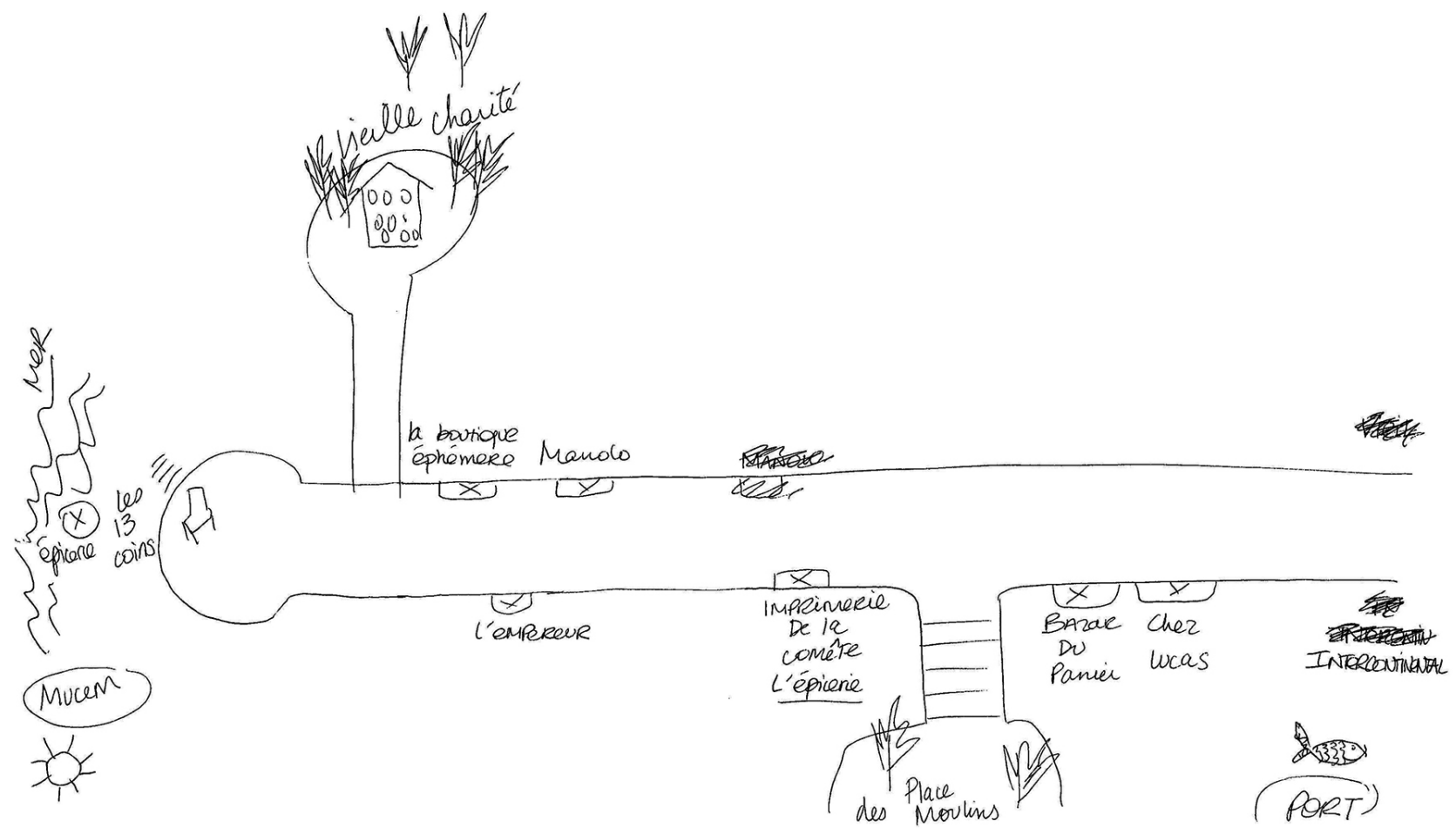

Figure 7 : Carte mentale d'une créatrice de vêtements du quartier du Panier Mental map of the Panier district by a local clothes designer

révèle l'importance des espaces de proximité tels que les quartiers urbains. Ces espaces sont encore animés de relations sociales, d'échanges économiques, de tensions, de mouvements d'appropriation et de revendications symboliques. En définitive, les résultats présentés dans ce texte incitent à considérer l'ancrage dans le quartier et les pratiques qui s'y inscrivent comme complémentaires de la mobilité des individus et de leurs échanges numériques.

\section{Bibliographie}

Ambrosino C., 2013. Portrait de l'artiste en créateur de ville. L'exemple du quartier artistique de South Shoreditch à Londres, Territoire en Mouvement, ${ }^{\circ}$ 17-18, p. 20-37.

Ambrosino C., 2009. Le cluster culturel, un artefact conceptuel pour mieux comprendre la ville contemporaine. L'exemple du quartier Berriat à Grenoble, in Vallat C. (dir.), Pérennité urbaine, ou la ville par-delà ses métamorphoses. Volume 1 Traces, Paris, L'Harmattan, p.59-70.

Attard-Maraninchi M.-F., 1997. Le Panier, village corse à Marseille, Paris, Autrement, 157 p.
Benoit-Guilbot O., 1986. Quartiers-dortoirs ou quartiers-villages?, in L'Esprit des lieux. Localités et changement social en France, Paris, CNRS, p.127-156.

Bidou-Zachariasen C., 1984. Les aventuriers du quotidien: essai sur les nouvelles classes moyennes, Paris, PUF, 200 p.

Boltanski L., Chiapello E., 1999. Le nouvel esprit du capitalisme, Paris, Gallimard, 843 p.

Boulllon F., 2003. Des migrants et des squats : précarités et résistances aux marges de la ville, Revue européenne des migrations internationales, $\mathrm{n}^{\circ} 2$, vol. 19, p. 23-46. DOI : http://doi.org/10.4000/remi.440.

Boulay G., 2011. Le prix de la ville. Le marché immobilier à usage résidentiel dans l'aire urbaine de Marseille-Aix-en-Provence (1990-2010). Thèse de doctorat en géographie, Université de Provence, 565 p.

Chantelot S., 2010. Vers une mesure de la créativité : la construction de la classe créative française, Revue d'Économie Régionale et Urbaine, $\mathrm{n}^{\circ}$ 3, p. 511-540.

Charmes E., 2005. Le retour à la rue comme support de la gentrification, Espaces et Sociétés, n ${ }^{\circ}$ 122, vol. 4, p. 115-135. DOI : http://doi.org/10.3917/esp.122.0115.

Charmes E., 2006. La rue, village ou décor? Parcours dans deux rues de Belleville, Grâne, Créaphis, 157 p.

Collet A., 2008. Les « gentrifieurs » du Bas-Montreuil : vie résidentielle et vie professionnelle, Espaces et sociétés, $n^{\circ} 132-133$, p. 125-141. 
Collet A., 2012. Le loft : habitat atypique et innovation sociale pour deux générations de «nouvelles classes moyennes », Espaces et sociétés, n ${ }^{\circ} 148-149$, p. 37-52.

Couvrand L., 2005. Les petites mains de l'usine LU, Nantes Au Quotidien, $\mathrm{n}^{\circ} 151$, p. 26-28.

Di MÉo G., 1994. Épistémologie des approches géographiques et socio-anthropologiques du quartier urbain. Annales de Géographie, no 577, vol. 103, p. 255-275. DOI : http://doi. org/10.3406/geo.1994.13781.

DouART P., 2008. La ville méditerranéenne: le renouvellement durable des éléments patrimoniaux dans un contexte de géogouvernance. Thèse de doctorat en géographie, Université de Provence, $529 \mathrm{p}$.

Emin S., Sagot-Duvauroux D., 2016. L'émergence de dynamiques coopératives : l'exemple d'un réseau d'entreprises créatives co-localisées. Une approche par l'économie des proximités. Géographie, Économie, Société, n ${ }^{4}$, vol. 18, p. 525-550. DOI : 10.3166/ges.18.525-550.

Garden M., Guillaume P., Lacave M., 1986. Introduction historique, in L'Esprit des lieux. Localités et changement social en France, Paris, CNRS, p.13-38.

Giesening J.J., 2013. Where We Go from Here: the Spatial Mental Mapping Method and Its Analytic Components for Social Science Data Gathering, Qualitative Inquiry, $\mathrm{n}^{\circ}$ 9, vol. 19, p. 712-724.

Giroud M., 2007. Résister en habitant? Renowvellement urbain et continuités populaires en centre ancien (Berriat SaintBruno à Grenoble et Alcântara à Lisbonne). Thèse de doctorat en géographie, Université de Poitiers, 518 p.

Giroud M., 2011. Usages des espaces rénovés et continuités populaires en centre ancien, Espaces et sociétés, n 144-145, p. $37-54$.

Iorio C., 2011. Normalisation de l'habitat. Entre protection des occupants et uniformisation des «modes d'habiter », Techniques \& Culture, $\mathrm{n}^{\circ}$ 56, p. 166-177.

Jacobs J., 1991. Déclin et survie des grandes villes américaines, Liège, Pierre Mardaga, 435 p.

Jourdan S., 2008. Un cas aporétique de gentrification : la ville de Marseille, Méditerranée, no 2, vol. 111, p. 85-90.

KEA-Commission EuropéEnne, 2006. The economy of culture in Europe, étude préparée pour l'UE, 355 p.

Le Goff J.-P., 2012. La fin du village. Une histoire française, Paris, Gallimard, 577 p.

Lévy J., Lussault M. (dir.), 2003. Dictionnaire de la géographie et de l'espace des sociétés, Paris, Belin, 1033 p.

Liefooghe C., 2010. Économie créative et développement des territoires : enjeux et perspectives de recherche, Innovations, $\mathrm{n}^{\circ}$ 31, vol. 1, p. 181-197.

LLoyd R., 2002. Neo-Bohemia : Art and Neighborhood Redevelopment in Chicago, Journal of Urban Affairs, $\mathrm{n}^{\circ}$ 5, vol. 24, p. 517 532. DOI : http://doi.org/10.1002/9780470752814.ch16.

Lubart T., 2003. Psychologie de la créativité, Paris, Armand Colin, $186 \mathrm{p}$.

Mendras H., 1995. Les sociétés paysannes : éléments pour une théorie de la paysannerie, Paris, Gallimard, 368 p.

Menger P.-M., 2002. Portrait de l'artiste en travailleur. Métamorphoses du capitalisme, Paris, Seuil, 96 p.
Mommans H., 2004. Cultural clusters and the post-industrial city: towards the remapping of urban cultural policy, Urban Studies, $\mathrm{n}^{\circ}$ 3, vol. 41, p. 507-532. DOI : 10.1080/0042098042000178663.

Moser G., Weiss K., 2003 (dir.). Espaces de vie. Aspects de la relation homme-environnement, Paris, Armand Colin, 396 p.

Nader B., 2012. Perception, appropriation et représentations des territoires de vie des 75 ans et plus dans le XVI e arrondissement parisien : l'apport des cartes mentales, in VIRIOTDurandal J.-P., Pihet C., Chapon P.-M. (dir.), Les défis territoriaux face au vieillissement, Paris, La Documentation française, p. 47-68.

Ovidio M., Ponzini D., 2014. Rhetoric and effects of the creative city policy : evidence and reflections from Milan and beyond, in Sternberg R., Krauss G. (dir.), Handbook of research on entrepreneurship and creativity, Cheltenham et Northampton, Edward Elgar, p. 296-322.

Pelras C., 2001. Goulien, commune bretonne du Cap Sizun. Entre XIX siècle et III ${ }^{e}$ millénaire, Rennes, PUR, 486 p.

Petiteau J.-Y., 2012. Nantes, récit d'une traversée. MadeleineChamp-de-Mars, Paris, Carré, 284 p.

Ramadier T., 2003. Les représentations cognitives de l'espace: modèles, méthodes et utilité, in Moser G., Weiss K. (dir.), Espaces de vie. Aspects de la relation homme-environnement, Paris, Armand Colin, p. 177-200.

Roy E., 2004. La mise en culture des friches urbaines. Territoires en transition à Nantes, Les Annales de La Recherche Urbaine, $\mathrm{n}^{\circ}$ 97, p. 121-126.

ScotT A.J., Leriche F., 2005. Les ressorts géographiques de l'économie culturelle : du local au mondial, L'Espace Géographique, n 3, vol. 34, p. 207-222.

Sмiтн N., 2003. La gentrification généralisée : d'une anomalie locale à la « régénération » urbaine comme stratégie urbaine globale, in Bidou-Zachariasen C. (dir.), Retours en ville, Paris, Descartes \& Cie, p. 45-72.

Sтоск M., 2006. L'hypothèse de l'habiter poly-topique : pratiquer les lieux géographiques dans les sociétés à individus mobiles. EspacesTemps.net, 26/02/2006, [http://www.espacestemps.net/articles/hypothese-habiter-polytopique/].

Torre A., Rallet A., 2005. Proximity and Localization, Regional Studies, ${ }^{\circ}$ 1, vol. 39, p. 47-59. DOI : 10.1080/0034340052000320842.

Tremblay G., 2008. Industries culturelles, économie créative et société de l'information, Global Media Journal - Édition Canadienne, $\mathrm{n}^{\circ}$ 1, vol. 1, p. 65-88.

Vivant E., 2009. Qu'est-ce que la ville créative?, Paris, PUF, $92 \mathrm{p}$.

Vivant E., Charmes E., 2008. La gentrification et ses pionniers : le rôle des artistes off en question, Métropoles, $\mathrm{n}^{\circ} 3$, p. 29-66.

Vivant E., Tremblay D.-G., 2010. L'économie créative. Revue des travaux francophones, note de recherche de la Chaire de recherche du Canada sur les enjeux socio-organisationnels de l'économie du savoir, Téluq-UQAM, nº 10-02, 71 p.

Wester P., 2005. Lorsque la rue des Olivettes était noire de monde, Nantes Au Quotidien, n' 160, p. 29-31. 